\title{
Abordagem bayesiana para avaliação da adaptabilidade e estabilidade de genótipos de alfafa
}

\author{
Moysés Nascimento(1), Fabyano Fonseca e Silva(1), Thelma Sáfadi(2), Ana Carolina Campana Nascimento(1), \\ Reinaldo de Paula Ferreira ${ }^{(3)}$ e Cosme Damião Cruz ${ }^{(4)}$
}

(1)Universidade Federal de Viçosa (UFV), Departamento de Estatística, Avenida P.H. Rolfs, s/no, CEP $36571-000$ Viçosa, MG. E-mail: moysesnascim@ufv.br, fabyanofonseca@ufv.br, ana.campana@ufv.br (2)Universidade Federal de Lavras, Departamento de Ciências Exatas, Setor de Estatística e Experimentação, Campus Universitário, Caixa Postal 3037, CEP 37200-000 Lavras, MG. E-mail: safadi@ufla.br ${ }^{(3)}$ Embrapa Pecuária Sudeste, Rodovia Washington Luiz, Km 234, CEP 13560-970 São Carlos, SP. E-mail: reinaldo@cppse.embrapa.br (4)UFV, Departamento de Biologia Geral. E-mail: cdcuz@ufv.br

\begin{abstract}
Resumo - O objetivo deste trabalho foi propor uma abordagem bayesiana do método de Eberhart \& Russell para avaliar a adaptabilidade e da estabilidade fenotípica de genótipos de alfafa (Medicago sativa), bem como avaliar a eficiência da utilização de distribuições a priori informativas e pouco informativas. Foram utilizados dados de um experimento em blocos ao acaso, no qual se avaliou a produção de massa de matéria seca de 92 genótipos. A metodologia bayesiana proposta foi implementada no programa livre R por meio da função MCMCregress do pacote MCMCpack. Para representar as distribuições a priori pouco informativas, utilizaram-se distribuições de probabilidade com grande variância; e, para representar distribuições a priori informativas, adotou-se o conceito de meta-análise, que se caracteriza pela utilização de informações provenientes de trabalhos anteriores. A comparação entre as distribuições a priori foi realizada por meio do fator de Bayes, com a função BayesFactor do pacote MCMCpack, que indicou a priori informativa como a mais adequada nas condições deste estudo.
\end{abstract}

Termos para indexação: Medicago sativa, fator de Bayes, priori informativa, interação genótipo x ambiente, MCMC.

\section{Bayesian approach for the evaluation of adaptability and stability of alfalfa genotypes}

\begin{abstract}
The objective of this work was to propose a Bayesian approach for the Eberhart \& Russell method to evaluate the phenotypic adaptability and stability of alfafa (Medicago sativa) genotypes, as well as to evaluate the efficiency of the use of prior informative and noninformative distributions. Data from a randomized block design experiment evaluating the forage dry weight of 92 genotypes were used. The Bayesian methodology proposed was implemented in the free software $\mathrm{R}$ by the MCMCregress function of the MCMCpack package. To represent the noninformative prior distributions, a probability distribution with high variance was used; and, to represent the informative prior, a meta-analysis concept was adopted, characterized by the use of information provided by previous studies. The comparison between the prior distributions was done using the Bayes Factor, with the BayesFactor function of the MCMCpack package, which indicated that an informative prior is more appropriate under the conditions of this study.
\end{abstract}

Index terms: Medicago sativa, Bayes factor, informative prior, genotype x environment interaction, MCMC.

\section{Introdução}

No melhoramento genético de plantas o estudo pormenorizado da interação entre genótipo x ambiente é de extrema importância, quando o objetivo é selecionar ou recomendar genótipos para o plantio. Diversas metodologias são usadas para esse fim, como o método de Annicchiarico (1992), que se baseia em análise da variância, e o método de Eberhart \& Russell (1966), que se baseia em análise de regressão linear simples. Métodos não paramétricos, como os desenvolvidos por Rocha et al. (2005) e Nascimento et al. (2009a, 2009b, 2010c), também podem ser empregados.

Apesar da grande quantidade de metodologias, nenhuma faz uso de informações a priori na estimação dos parâmetros. A inserção de informações prévias pode ser realizada por meio de inferência bayesiana. Nesta abordagem, a incerteza sobre os parâmetros de

Pesq. agropec. bras., Brasília, v.46, n.1, p.26-32, jan. 2011 
interesse é representada por meio de distribuições de probabilidade específicas, que retratam o maior ou menor conhecimento sobre os parâmetros.

O método de Eberhart \& Russell (1966) é de grande utilidade e possibilita que a abordagem bayesiana seja realizada sem grandes dificuldades. Embora não seja recente, esse método é de extrema importância e bastante relatado na literatura especializada, como em Ferreira et al. (2004), que estudaram a adaptabilidade e a estabilidade de 35 genótipos de alfafa, em Lédo et al. (2005), que avaliaram a adaptabilidade e estabilidade de cultivares de alfafa cultivadas em diferentes regiões do Estado de Minas Gerais, e em Vasconcelos et al. (2008), que avaliaram e selecionaram genótipos de alfafa por meio de metodologias de adaptabilidade e estabilidade fenotípica. Miranda et al. (2009) fizeram uso da metodologia de Eberhart \& Russell (1966) para corroborar os resultados de duas técnicas de análise de adaptabilidade e estabilidade multivariadas em milho-pipoca. Pereira et al. (2009) compararam métodos de análise de adaptabilidade e estabilidade fenotípica em feijoeiro-comum.

O objetivo deste trabalho foi apresentar uma abordagem bayesiana do método de Eberhart \& Russell para análise da adaptabilidade e da estabilidade fenotípica de genótipos de alfafa (Medicago sativa L.), e avaliar a eficiência da utilização de distribuições a priori informativas e pouco informativas.

\section{Material e Métodos}

Foram utilizados dados de um experimento conduzido pela Embrapa Pecuária Sudeste, para o desenvolvimento de genótipos de alfafa adaptados a diferentes ecossistemas brasileiros. Neste experimento, que utilizou o delineamento de blocos ao acaso com duas repetições, foi avaliada a produção de massa de matéria seca de 92 genótipos de alfafa, submetidos a 20 cortes no período de novembro de 2004 a junho de 2006. Os cortes foram caracterizados como diferentes condições ambientais, porque foram realizados em épocas diferentes.

Para a análise bayesiana, foram considerados apenas os genótipos avaliados em ao menos dois dos seguintes trabalhos: Ferreira et al. (2004), Lédo et al. (2005) e Vasconcelos et al. (2008), utilizados como referência para a especificação das distribuições a priori.

O método proposto por Eberhart \& Russell (1966) baseia-se na análise de regressão linear simples, que mede a resposta de cada genótipo às variações ambientais. Para um experimento com g genótipos, a ambientes e $r$ repetições, define-se o seguinte modelo estatístico:

$$
Y_{\mathrm{ij}}=\beta_{0 \mathrm{i}}+\beta_{\mathrm{li}} \mathrm{I}_{\mathrm{j}}+\psi_{\mathrm{ij}} \text {, }
$$

em que: $Y_{\mathrm{ij}}$, média do genótipo $\mathrm{i}$ no ambiente $\mathrm{j}$; $\beta_{0 \mathrm{i}}$, coeficiente linear referente ao i-ésimo genótipo; $\beta_{\mathrm{li}}$, coeficiente de regressão, que mede a resposta do i-ésimo genótipo à variação do ambiente;

$\mathrm{I}_{\mathrm{j}}$, índice ambiental codificado, $\mathrm{I}_{\mathrm{j}}=\left(\frac{\sum_{\mathrm{j}} \mathrm{Y}_{\mathrm{j}}}{\mathrm{g}}-\frac{\sum_{\mathrm{i}} \sum_{\mathrm{j}} \mathrm{Y}_{\mathrm{j}}}{\mathrm{ga}}\right)$;

$\psi_{\mathrm{ij}}$, erros aleatórios, que podem ser descompostos como: $\psi_{\mathrm{ij}}=\delta_{\mathrm{ij}}+\bar{\varepsilon}_{\mathrm{ij}}$, em que: $\delta_{\mathrm{ij}}$, desvio da regressão, e $\bar{\varepsilon}_{\mathrm{ij}}$, erro experimental médio.

As estimativas de $\mathrm{I}_{\mathrm{j}}$ indicam a qualidade do ambiente, em que valores negativos de $\mathrm{I}_{\mathrm{j}}$ identificam ambientes desfavoráveis, e valores positivos de $\mathrm{I}_{\mathrm{j}}$, ambientes favoráveis.

Os parâmetros de adaptabilidade são dados por:

$\bar{\beta}_{0 \mathrm{j}}=\mathrm{Y}_{\mathrm{i}}$ e $\bar{\beta}_{\mathrm{li}}=\frac{\Sigma_{\mathrm{j}} \mathrm{Y}_{\mathrm{ij}} \mathrm{I}_{\mathrm{j}}}{\sum_{\mathrm{j}} \mathrm{I}_{\mathrm{j}}^{2}}$, enquanto o de estabilidade é dado por: $\hat{\sigma}_{\mathrm{d}_{\mathrm{i}}}^{2}=\frac{\mathrm{QMD}_{\mathrm{i}}-\mathrm{QMR}}{\mathrm{r}}$, em que $\mathrm{QMD}_{\mathrm{i}}$ é o quadrado médio do desvio da regressão de cada genótipo e QMD, o quadrado médio do resíduo fornecido pela análise da variância (Cruz et al., 2004).

$\mathrm{Na}$ abordagem frequentista, em geral empregada, as hipóteses de interesse, $\mathrm{H}_{0}: \beta_{\mathrm{li}}=1$ versus $\mathrm{H}_{1}: \beta_{\mathrm{li}} \neq 1$ e $\mathrm{H}_{0}: \sigma_{\mathrm{d}_{\mathrm{i}}}^{2}=0$ versus $\mathrm{H}_{1}: \sigma_{\mathrm{d}_{\mathrm{i}}}^{2}>0$, são avaliadas pelas estatísticas t e F, respectivamente.

$\mathrm{Na}$ abordagem bayesiana, fundamentada no teorema de Bayes - posteriori $\propto$ verossimilhança x priori - toda informação a respeito dos parâmetros a serem estimados está contida na distribuição a posteriori, que é composta de informações prévias sobre os parâmetros, representadas pela distribuição a priori, e de informações contidas nos dados a serem analisados, representadas pela função de verossimilhança.

Ao se considerar o modelo estatístico $\mathrm{Y}_{\mathrm{ij}}=\beta_{0 \mathrm{i}}+\beta_{\mathrm{li}} \mathrm{I}_{\mathrm{j}}+\psi_{\mathrm{ij}}$ e assumir-se que cada observação $\mathrm{Y}_{\mathrm{ij}}$ tem distribuição $\mathrm{Y}_{\mathrm{ij}} \sim \mathrm{N}\left(\beta_{0 \mathrm{i}}+\beta_{\mathrm{li}} \mathrm{I}_{\mathrm{j}} \sigma_{\mathrm{i}}^{2}\right)$, a função de verossimilhança para cada genótipo i é dada por:

$$
\begin{aligned}
& \mathrm{L}_{\mathrm{i}}\left(\beta_{0 \mathrm{i}}, \beta_{\mathrm{li}}, \sigma_{\mathrm{i}}^{2} ; \mathrm{y}_{\mathrm{ij}}\right)=\prod_{\mathrm{j}=1}^{\mathrm{a}=1} \frac{1}{\sqrt{2} \pi \sigma_{\mathrm{i}}^{2}} \exp \left\{-\frac{1}{2 \sigma_{\mathrm{i}}^{2}}\left[\mathrm{y}_{\mathrm{ij}}-\left(\beta_{0 \mathrm{i}}+\beta_{\mathrm{li}} \mathrm{I}_{\mathrm{j}}\right)^{2}\right]\right\} \\
& =\frac{1}{\left(\sqrt{2} \pi \sigma_{\mathrm{i}}^{2}\right)^{\mathrm{a}}} \exp \left\{-\frac{1}{2 \sigma_{\mathrm{i}}^{2}} \sum_{\mathrm{j}=1}^{\mathrm{a}}\left[\mathrm{y}_{\mathrm{ij}}-\left(\beta_{0 \mathrm{i}}+\beta_{\mathrm{li}} \mathrm{I}_{\mathrm{j}}\right)^{2}\right]\right\}, \forall \mathrm{i} .
\end{aligned}
$$


Para a estimação dos parâmetros de adaptabilidade e estabilidade, é necessário atribuir distribuições a priori para os parâmetros. Para $\beta_{0 \mathrm{i}}$, $\beta_{\mathrm{li}}$ e $\sigma^{2}$ foram consideradas as seguintes distribuições: $\beta_{0 \mathrm{i}} \sim \mathrm{N}\left(\mu_{0 \mathrm{i}}, \sigma_{0 \mathrm{i}}^{2}\right), \beta_{\mathrm{li}} \sim \mathrm{N}\left(\mu_{\mathrm{li}}, \sigma_{\mathrm{li}}^{2}\right), \sigma_{\mathrm{i}}^{2} \sim \operatorname{GamaInv}\left(\alpha_{\mathrm{i}} \beta_{\mathrm{i}}\right)$; esta última uma gama inversa com média e variância iguais a

\section{$\frac{\beta_{i}}{\alpha_{i}-1}$ e $\frac{\beta_{i}^{2}}{\left(\alpha_{i}-1\right)^{2}\left(\alpha_{i}-2\right)}$, respectivamente.}

Ao assumir-se a independência entre os parâmetros dessas distribuições, a priori conjunta para cada genótipo é dada por:

$$
\begin{aligned}
& P_{\mathrm{i}}\left(\beta_{0 \mathrm{i}}, \beta_{\mathrm{li}}, \sigma_{\mathrm{i}}^{2}\right)=\frac{1}{\sqrt{2} \pi \sigma_{0 \mathrm{i}}^{2}} \exp \left\{-\frac{1}{2 \sigma_{0 \mathrm{i}}^{2}}\left(\beta_{0 \mathrm{i}}-\mu_{0 \mathrm{i}}\right)^{2}\right\} \times \frac{1}{\sqrt{2} \pi \sigma_{\mathrm{li}}^{2}} \\
& \exp \left\{-\frac{1}{2 \sigma_{\mathrm{li}}^{2}}\left(\beta_{\mathrm{li}}-\mu_{\mathrm{li}}\right)^{2}\right\} \times \frac{1}{\left(\beta_{\mathrm{i}}^{\alpha_{\mathrm{i}}} \mathrm{G}\left(\alpha_{\mathrm{i}}\right)\right.}\left(\frac{1}{\sigma_{\mathrm{i}}^{2}}\right)^{\alpha_{\mathrm{i}}+1} \exp \left\{-\frac{1}{\beta_{\mathrm{i}} \sigma_{\mathrm{i}}^{2}}\right\} \\
& \propto \exp \left\{-\frac{1}{2 \sigma_{\mathrm{i}}^{2}}\left(\beta_{0 \mathrm{i}}-\mu_{0 \mathrm{i}}\right)^{2}\right\} \times \frac{1}{\sqrt{2} \pi \sigma_{\mathrm{li}}^{2}} \exp \left\{-\frac{1}{2 \sigma_{\mathrm{li}}^{2}}\left(\beta_{\mathrm{li}}-\mu_{\mathrm{li}}\right)^{2}\right\} \\
& \times\left(\frac{1}{\sigma_{\mathrm{i}}^{2}}\right)^{\alpha_{\mathrm{i}}+1} \exp \left\{-\frac{1}{\beta_{\mathrm{i}} \sigma_{\mathrm{i}}^{2}}\right\}
\end{aligned}
$$

Para fazer inferências sobre os parâmetros de interesse, énecessário obter suas distribuições marginais a posteriori. Ao denotar o vetor de parâmetros para cada genótipo i por $\theta_{\mathrm{pi}}=\left(\beta_{\mathrm{li}}, \beta_{2 \mathrm{i}}, \sigma_{3 \mathrm{i}}^{2}\right)$, em que $\mathrm{p}=1,2,3$, a distribuição marginal a posteriori para o parâmetro $\theta_{\mathrm{pi}}$ é obtida pela seguinte integral: $\mathrm{P}\left(\theta_{\mathrm{pi}} \mid \mathrm{x}\right)=\int \mathrm{P}\left(\theta_{\mathrm{i}} \mid \mathrm{x}\right) \mathrm{d} \theta_{\text {.pi }}$, isto é, a integral em relação a todos os parâmetros do vetor, exceto o p-ésimo componente.

Na maioria dos casos, essas integrais são complexas e não apresentam soluções exatas. Para contornar esse problema, utiliza-se outra metodologia. Obtém-se uma amostra da distribuição conjunta a posteriori ao se utilizar as cadeias de Markov e o método de Monte Carlo, para a obtenção dos momentos de interesse das distribuições marginais (Cassela \& George, 1992). Neste estudo, a metodologia foi implementada no programa R (R Development Core Team, 2010), e a amostra da distribuição conjunta foi obtida por meio da função MCMCregress do pacote MCMCpack.

Para avaliar a influência de informações a priori na estimação dos parâmetros de adaptabilidade e estabilidade, optou-se pela utilização de dois modelos diferentes.

No Modelo 1, foram consideradas distribuições a priori informativas, cujas informações advém da aplicação da técnica de meta-análise, que se caracteriza pela utilização de informações de trabalhos anteriores: Ferreira et al. (2004), Lédo et al. (2005) e Vasconcelos et al. (2008). As informações foram inseridas na análise por meio dos valores assumidos para os parâmetros das distribuições a priori, denominados de hiperparâmetros.

Estes valores foram dados pela média e variância da amostra composta pelas estimativas dos parâmetros obtidas das referências citadas (Tabela 1), o que resultou nas distribuições: $\beta_{0 \mathrm{i}} \sim \mathrm{N}\left(\mu_{0 \mathrm{i}}=\bar{\beta}_{0 \mathrm{i}}, \sigma_{\mathrm{li}}^{2}=\operatorname{Var}\left(\bar{\beta}_{0 \mathrm{i}}\right)\right)$, $\beta_{\mathrm{li}} \sim \mathrm{N}\left(\mu_{\mathrm{li}}=\bar{\beta}_{\mathrm{li}}, \sigma_{\mathrm{li}}^{2}=\operatorname{Var}\left(\bar{\beta}_{1}\right)\right)$ e $\sigma_{\mathrm{i}}^{2} \sim \operatorname{GamaInv}\left(\alpha_{1}, \beta_{\mathrm{i}}\right)$, em que: $\bar{\beta}_{0 \mathrm{i}}$, médias das estimativas dos $\beta_{0 \mathrm{i}} ; \bar{\beta}_{\mathrm{li}}$, médias das estimativas dos $\beta_{\text {li; }}$, $\operatorname{Var}\left(\bar{\beta}_{0 \mathrm{i}}\right)$, variância dos valores médios de $\bar{\beta}_{0 i} ; \operatorname{Var}\left(\bar{\beta}_{\mathrm{li}}\right)$, variância dos valores médios de $\beta_{\text {li; }} ; \alpha_{\mathrm{i}}$ e $\beta_{\mathrm{i}}$, valores obtidos pela resolução do sistema:

$$
\left\{\begin{array}{c}
\bar{\sigma}_{i}^{2}=\frac{\beta_{i}}{\alpha_{i}-1} \\
\operatorname{Var}\left(\bar{\sigma}_{i}^{2}\right)=\frac{\beta_{i}^{2}}{\left(\alpha_{i}-1\right)^{2}\left(\alpha_{i}-2\right)}, \text { as quais foram: } \\
\alpha_{i}=\frac{\left(\sigma_{i}^{2}\right)^{3}}{\operatorname{Var}\left(\bar{\sigma}_{i}^{2}\right)^{*} \bar{\sigma}_{i}^{2}} \beta_{i}=\frac{\left(\sigma_{i}^{2}\right)^{3}}{\operatorname{Var}\left(\bar{\sigma}_{i}^{2}\right)+\bar{\sigma}_{i}^{2}}
\end{array}\right.
$$

Dos 92 genótipos avaliados no experimento, apenas 12 foram avaliados em ao menos dois dos trabalhos usados como referência para a especificação da priori. Por isso, somente os 12 genótipos apresentados na Tabela 2 foram considerados para a análise bayesiana.

No Modelo 2, foram utilizadas distribuições a priori pouco informativas, que representam distribuições de probabilidade com grande variância. Foram adotadas as seguintes distribuições: $\beta_{0 \mathrm{i}} \sim \mathrm{N}\left(\mu_{0 \mathrm{i}}=0, \sigma_{0 \mathrm{i}}^{2}=1000000\right)$, $\beta_{\mathrm{li}} \sim \mathrm{N}\left(\mu_{\mathrm{li}}=0, \sigma_{\mathrm{li}}^{2}=1000000\right)$ e $\sigma_{\mathrm{i}}^{2} \sim \operatorname{GamaInv}\left(\alpha_{\mathrm{l}}=0,00001\right.$; $\left.\beta_{\mathrm{i}}=5000\right)$. A Figura 1 apresenta as distribuições pouco informativas obtidas por meio dos hiperparâmetros fornecidos.

Tabela 1. Análise da variância para a produção de matéria seca, ao avaliar-se 20 genótipos de alfafa no período de novembro de 2004 a junho de 2006.

\begin{tabular}{lcc}
\hline Fonte de variação & GL & Quadrados médios \\
\hline Blocos & 1 & $2.002 .415,43$ \\
Cultivar (Cv) & 91 & $1.384 .475,75^{*}$ \\
Erro a & 91 & $574.269,72$ \\
Corte (Co) & 91 & $62.331 .022,56^{*}$ \\
Erro b & 91 & $946.917,67$ \\
Interação (Ge x Co) & 1.729 & $60.682,46^{* *}$ \\
Erro c & 1.729 & $55.851,26$ \\
\hline CV a (\%) & 64.39 & - \\
CV b (\%) & 82,69 & - \\
CV c (\%) & 20,08 & - \\
Média & 1.176 & - \\
\hline
\end{tabular}

ns Não significativo. ${ }^{* *}$ e *Significativo a 5 e $1 \%$ de probabilidade, respectivamente, pelo teste $\mathrm{F}$. 
A comparação entre os modelos 1 e 2, ou seja, entre distribuições a priori informativas e não informativas, foi realizada com base no fator de Bayes (Kass \& Raftery, 1995). O cálculo do fator de Bayes foi realizado por meio da função BayesFactor do pacote MCMCpack.

De acordo com Jeffreys (1961), o fator de Bayes pode ser interpretado da seguinte forma: $\mathrm{FB}_{\mathrm{ij}}<1$ demonstra evidência a favor do modelo $\mathrm{j} ; 1 \leq \mathrm{FB}_{\mathrm{ij}}<3$ demonstra evidência moderada a favor do modelo $\mathrm{i} ; 3 \leq \mathrm{FB}_{\mathrm{ij}}<10$ demonstra evidência substancial a favor do modelo i;
$10 \leq \mathrm{FB}_{\mathrm{ij}}<30$ demonstra evidência forte a favor de $\mathrm{i}$; $30 \leq \mathrm{FB}_{\mathrm{ij}}<100$ demonstra evidência muito forte a favor de $\mathrm{i}$; e $\mathrm{FB}_{\mathrm{ij}} \geq 100$ demonstra evidência decisiva a favor do modelo $\mathrm{i}$.

Com relação ao parâmetro de estabilidade $\left(\sigma_{\mathrm{di}}^{2}\right)$, as amostras de sua distribuição marginal foram obtidas indiretamente, uma vez que esse parâmetro representa uma função de $\sigma_{i}^{2}$. Ao se obter valores para $\sigma_{i}^{2}$ indiretamente em cada iteração, obtém-se valores para $\sigma_{\mathrm{di}}^{2}$ por meio da seguinte expressão: $\hat{\sigma}_{\mathrm{di}}^{2}=\hat{\sigma}_{\mathrm{i}}^{2}-(\mathrm{QMR} / \mathrm{r})$, em que: QMR, quadrado médio do resíduo fornecido

Tabela 2. Estimativas e média da adaptabilidade e da estabilidade obtidas por meio da metodologia de Eberhart \& Russel (1966) em Ferreira et al. (2004), Lédo et al. (2005) e Vasconcelos et al. (2008).

\begin{tabular}{|c|c|c|c|c|c|c|c|c|c|c|c|c|}
\hline \multirow[t]{2}{*}{ Genótipo } & \multicolumn{3}{|c|}{ Ferreira et al. (2004) } & \multicolumn{3}{|c|}{ Lédo et al. (2005) } & \multicolumn{3}{|c|}{ Vasconcelos et al. (2008) } & \multicolumn{3}{|c|}{ Média } \\
\hline & $\beta_{0 \mathrm{i}}$ & $\beta_{\mathrm{li}}$ & $\sigma_{\mathrm{di}}^{2}$ & $\beta_{0 \mathrm{i}}$ & $\beta_{\mathrm{li}}$ & $\sigma_{\mathrm{di}}^{2}$ & $\beta_{0 \mathrm{i}}$ & $\beta_{\mathrm{li}}$ & $\sigma_{\mathrm{di}}^{2}$ & $\bar{\beta}_{0 \mathrm{i}}$ & $\bar{\beta}_{\mathrm{li}}$ & $\bar{\sigma}_{\mathrm{di}}^{2}$ \\
\hline Monarca SP INTA & $1.753,00$ & 1,09 & 0,00 & $1.216,00$ & 0,95 & $66.133,00$ & - & - & - & $1.484,50$ & 1,02 & $33.066,50$ \\
\hline Crioula & $1.587,00$ & 0,91 & $55.879,10$ & $1.627,00$ & 1,06 & $21.058,00$ & $1.745,00$ & 0,74 & $118.739,03$ & $1.653,00$ & 0,91 & $65.225,38$ \\
\hline 5929 & $1.587,90$ & 0,88 & $4.832,70$ & $1.052,00$ & 0,76 & $24.243,00$ & - & - & - & $1.319,95$ & 0,82 & $14.537,85$ \\
\hline $58 \mathrm{~N} 58$ & $1.714,60$ & 0,96 & 0,00 & $1.026,00$ & 0,63 & 0,00 & - & - & - & $1.370,30$ & 0,80 & 0,00 \\
\hline Sutter & $1.527,80$ & 0,98 & $18.541,70$ & $1.086,00$ & 1,08 & $57.364,00$ & - & - & - & $1.306,90$ & 1,03 & $37.952,85$ \\
\hline Flórida 77 & $1.691,50$ & 1,04 & $24.237,80$ & $1.231,00$ & 1,07 & $68.721,00$ & - & - & - & $1.461,25$ & 1,06 & $46.479,40$ \\
\hline WL 516 & $1.544,90$ & 0,85 & $8.715,80$ & $1.103,00$ & 0,56 & 0,00 & - & - & - & $1.323,95$ & 0,71 & $4.357,90$ \\
\hline Maricopa & $1.631,50$ & 1,05 & 0,00 & $1.003,00$ & 0,63 & $91.992,00$ & - & - & - & $1.317,25$ & 0,84 & $45.996,00$ \\
\hline Costera SP INTA & $1.488,90$ & 1,19 & $1.272,60$ & - & - & - & $1.045,93$ & 1,27 & $24.777,34$ & $1.267,42$ & 1,23 & $13.024,97$ \\
\hline Р 30 & $1.476,70$ & 1,08 & $34.100,10$ & $1.598,00$ & 1,10 & $57.282,00$ & $1.800,76$ & 1,04 & $37.659,95$ & $1.625,15$ & 1,07 & $43.014,02$ \\
\hline P 5715 & $1.720,70$ & 0,84 & $27.009,20$ & $1.254,00$ & 0,63 & 0,00 & $1.597,47$ & 0,97 & 0,00 & $1.524,06$ & 0,81 & $9.003,07$ \\
\hline Alfa 200 & $1.644,40$ & 0,90 & 0,00 & 1,04 & 0,95 & $28.753,00$ & - & - & - & 822,72 & 0,93 & $14.376,50$ \\
\hline
\end{tabular}

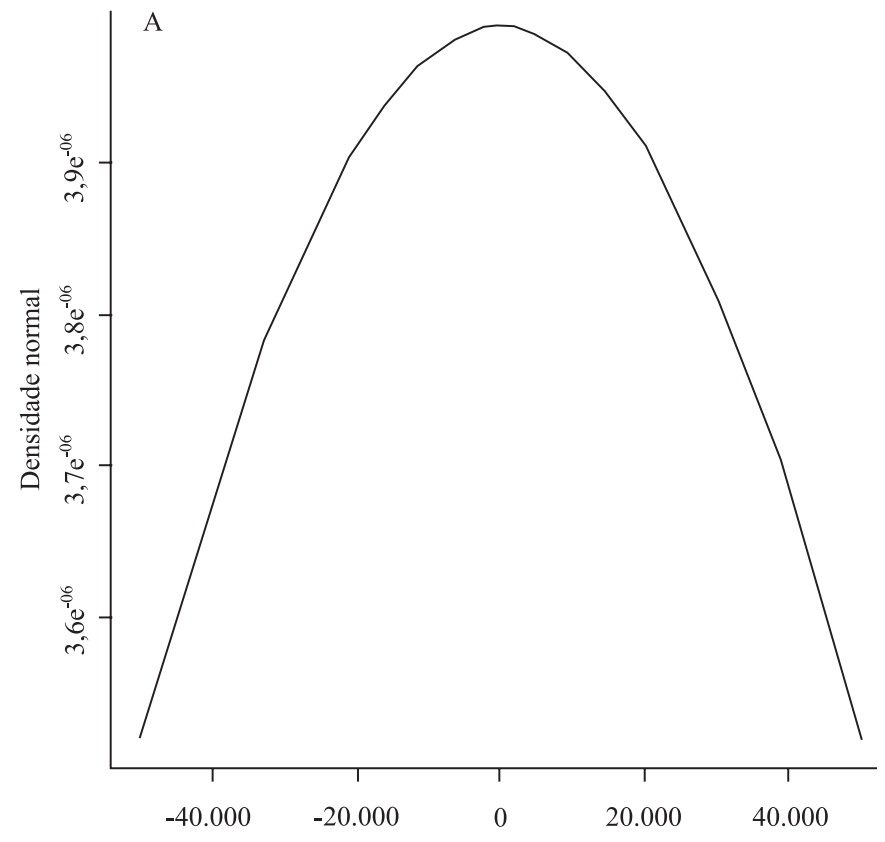

Parâmetro de adaptabilidade

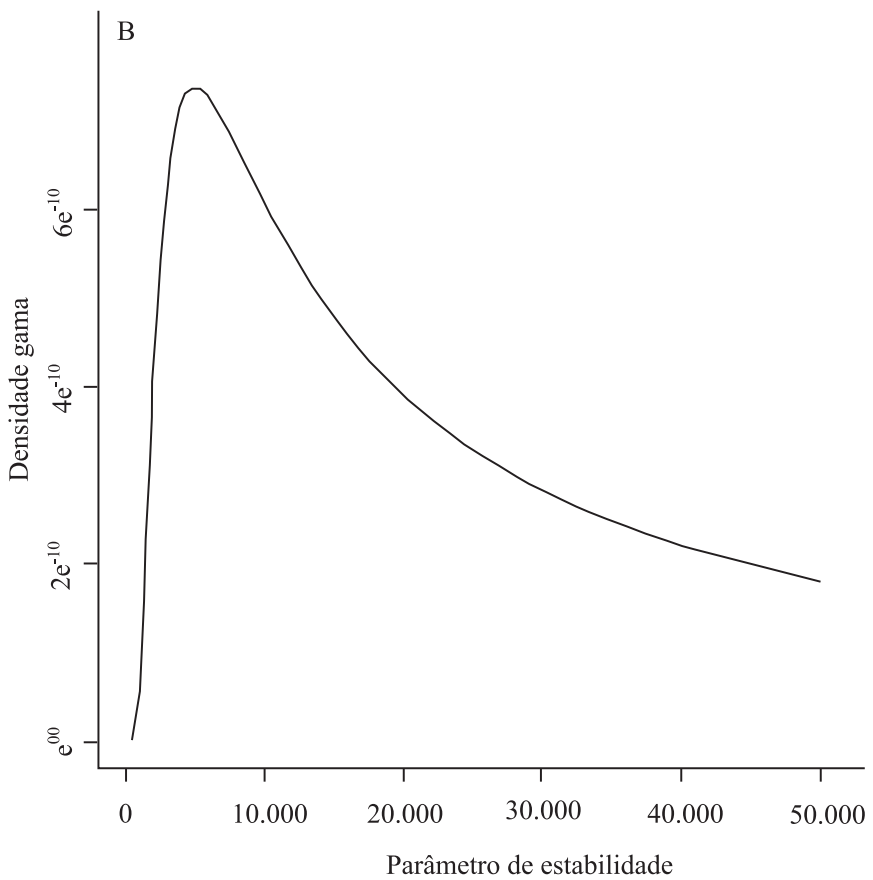

Parâmetro de estabilidade

Figura 1. Representação gráfica de distribuições pouco informativas utilizadas como informação a priori. A, normal $\left(\mu=0\right.$ e $\sigma_{d}^{2}$ $=100000)$; , gama inversa $(\alpha=0,00001$ e $\beta=5000)$. 
pela análise da variância e $\mathrm{r}$, número de repetições do experimento.

As hipóteses de interesse foram testadas pela construção de intervalos de credibilidade para os parâmetros; os intervalos foram obtidos diretamente da distribuição marginal a posteriori dos parâmetros. Assim, seja $\theta_{\mathrm{i}}=\left(\beta_{0 \mathrm{i}} \beta_{\text {li: }} \sigma_{\mathrm{d}_{\mathrm{i}}}^{2}\right)$ o vetor de parâmetros, o intervalo de credibilidade para $\theta_{\mathrm{i}}$, com probabilidade de cobertura $\delta$, é dado por:

$\int_{-\infty}^{\theta_{*}} \mathrm{P}_{\mathrm{i}}\left(\theta_{\mathrm{i}}=\left(\beta_{0 \mathrm{i}:} \beta_{\mathrm{li}:} \sigma_{{ }_{\mathrm{di}}}^{2}\right) \mid \mathrm{y}_{\mathrm{ij}}\right) \mathrm{d} \theta_{\mathrm{i}}=\alpha / 2 ;$

$\int_{\theta^{*}}^{-\infty} \mathrm{P}_{\mathrm{i}}\left(\theta_{\mathrm{i}}=\left(\beta_{0 \mathrm{i}} \beta_{\mathrm{li}:} \sigma_{\mathrm{di}_{\mathrm{i}}}^{2}\right) \mathrm{y}_{\mathrm{ij}}\right) \mathrm{d} \theta_{\mathrm{i}}=\alpha / 2$,

em que: $\theta_{*}$ e $\theta^{*}$ representam, respectivamente, os limites inferiores e superiores do intervalo de credibilidade.

Como o Gibbs sampler é um algoritmo iterativo, fazse necessário a verificação de sua convergência, que foi constatada, neste trabalho, por meio da aplicação dos critérios de Heidelberger \& Welch (1983), Raftery \& Lewis (1992) e Geweke (1992), implementados no pacote BOA (Bayesian Output Analysis) do programa R (R Development Core Team, 2010).

$\mathrm{Na}$ análise bayesiana de adaptabilidade e estabilidade, para cada parâmetro do modelo de regressão adotado, foram consideradas 110.000 iterações noalgoritmo Gibbs sampler, com um período de aquecimento ("burn-in") de 10.000 iterações. Para obter-se uma amostra não correlacionada, considerou-se um espaçamento entre os pontos amostrados de duas iterações ("thinning"), o que resultou em uma amostra final de tamanho 50.000. As amostras representam amostras das distribuições marginais a posteriori de cada parâmetro, sob as quais foi feita a inferência de cada parâmetro.

\section{Resultados e Discussão}

A Tabela 1 apresenta a análise da variância para a produção de massa de matéria seca. Os resultados mostram a significância da interação entre genótipos $\mathrm{x}$ cortes, o que indica que estes genótipos apresentaram desempenho diferenciado sob diferentes condições ambientais. Por causa dessas variações, foi necessário um estudo pormenorizado do comportamento dos genótipos por meio da análise de adaptabilidade e estabilidade.

Em relação à convergência, para todas as cadeias simuladas, o fator de dependência de Raftery \& Lewis
(1992) forneceu valores menores que cinco, e o p-valor do critério de Geweke (1992) foi sempre maior que o nível de significância pré-fixado (5\%), ou seja, ambos os critérios indicaram a convergência das cadeias geradas pelo Gibbs sampler. Para confirmar essa convergência, também utilizou-se o critério de Heidelberger \& Welch (1983), que verifica se os valores das cadeias são provenientes de uma distribuição estacionária.

Os valores das estimativas dos parâmetros de adaptabilidade e estabilidade foram obtidos pelo cálculo da média a posteriori, que são apresentadas, juntamente com os seus respectivos intervalos de credibilidade, na Tabela 3. No Modelo 1 (prioris informativas), dos 12 genótipos avaliados por meio da metodologia bayesiana, apenas o genótipo Costera SP INTA foi classificado como de adaptabilidade específica a ambientes favoráveis $\left(\beta_{1 i}>1\right)$, por causa dos limites do intervalo de credibilidade de $95 \%$. Os demais genótipos foram classificados como de adaptabilidade geral, já que o valor 1 pertence aos IC de $95 \%$. Em relação à estabilidade, somente o genótipo WL 516 foi classificado como de baixa estabilidade (previsibilidade), uma vez que o valor zero pertence ao IC de $95 \%$ para $\sigma_{\mathrm{di}}$.

A análise dos genótipos por meio do Modelo 2 (prioris não informativas) indicou que apenas o genótipo Flórida 77 foi de adaptabilidade específica a ambientes favoráveis, enquanto os demais foram de adaptabilidade geral. Como no Modelo 1, somente o genótipo WL 516 teve a hipótese de estabilidade rejeitada. Os critérios usados para a classificação destes genótipos foram os mesmos considerados ao se utilizar o modelo 1, isto é, os limites do IC de $95 \%$.

Ao se comparar as estimativas dos parâmetros obtidas pelos dois modelos, as estimativas do intercepto e do coeficiente angular foram semelhantes para cada genótipo. Houve apenas uma inversão de resultados; no Modelo 1, o genótipo Costera SP INTA foi considerado como adaptável a ambientes favoráveis, e de adaptabilidade geral no Modelo 2. A mesma inversão ocorreu para o genótipo Flórida, ao ser analisado pelo Modelo 2. Nesse modelo, três dos 12 genótipos avaliados tiveram seus intervalos de credibilidade com limite inferior exatamente igual a 1 , o que pode gerar dúvida quanto à classificação do cultivar. É necessário informar qual dos dois modelos apresentou maior qualidade de ajuste; e a resposta é dada pelo cálculo do fator de Bayes.

Na Tabela 4, são apresentados os valores fornecidos pelo fator de Bayes, que indicam que o Modelo 1

Pesq. agropec. bras., Brasília, v.46, n.1, p.26-32, jan. 2011 
Tabela 3. Estimativas da média a posteriori e intervalos de credibilidade (95\%) dos parâmetros de adaptabilidade e estabilidade, ao considerar-se prioris informativas e não informativas.

\begin{tabular}{|c|c|c|c|c|c|c|c|c|c|c|}
\hline Genótipo & $\bar{\beta}_{0 \mathrm{i}}$ & $\mathrm{LI} \bar{\beta}_{0 \mathrm{i}}$ & $\operatorname{LS} \bar{\beta}_{0 \mathrm{i}}$ & $\mathrm{LI} \bar{\beta}_{\mathrm{li}}$ & $\bar{\beta}_{\mathrm{li}}$ & $\mathrm{LS} \bar{\beta}_{\mathrm{li}}$ & $\bar{\sigma}_{i}^{2}$ & LI $\bar{\sigma}_{\mathrm{d}_{\mathrm{i}}}^{2}$ & $\bar{\sigma}_{d_{i}}^{2}$ & LS $\bar{\sigma}_{\mathrm{d}_{\mathrm{i}}}^{2}$ \\
\hline & \multicolumn{10}{|c|}{ Prioris informativas } \\
\hline Monarca SP INTA & $1.192,75$ & $1.192,75$ & $1.249,17$ & 0,95 & 1,04 & 1,13 & $1.6220,32$ & $-19.195,00$ & $-11.705,31$ & $1.712,00$ \\
\hline Crioula & $1.048,82$ & $1.048,82$ & $1.130,01$ & 0,92 & 1,05 & 1,18 & $3.2800,14$ & $-8.570,00$ & $4.874,51$ & $27.341,00$ \\
\hline 5929 & $1.265,00$ & $1.265,00$ & $1.316,86$ & 0,83 & 0,92 & 1,01 & $1.3630,00$ & $-20.839,00$ & $14.298,35$ & $-1.968,00$ \\
\hline $58 \mathrm{~N} 58$ & $1.264,69$ & $1.264,69$ & $1.311,14$ & 0,99 & 1,07 & 1,15 & $1.1191,02$ & $-22.196,00$ & $-16.734,61$ & $-6.583,00$ \\
\hline Sutter & $1.214,00$ & $1.214,00$ & $1.287,35$ & 0,87 & 0,99 & 1,11 & $2.8370,00$ & $-12.350,00$ & 445,84 & $23.179,00$ \\
\hline Flórida 77 & $1.291,70$ & $1.291,70$ & $1.365,12$ & 0,99 & 1,11 & 1,23 & $2.8058,21$ & $-12.199,00$ & 132,58 & $21.663,00$ \\
\hline WL 516 & $1.332,00$ & $1.332,00$ & $1.604,00$ & 0,42 & 0,71 & 1,00 & $555.800,00$ & $265.892,00$ & $527.918,70$ & $1.011 .033,00$ \\
\hline Maricopa & $1.456,36$ & $1.456,36$ & $1.531,25$ & 0,88 & 1,01 & 1,13 & $29.881,20$ & $-11.166,50$ & $1.955,57$ & $24.841,50$ \\
\hline Costera SP INTA & $1.355,91$ & $1.355,91$ & $1.418,41$ & 1,01 & 1,12 & 1,23 & $20.603,90$ & $-17.175,00$ & $-7.321,74$ & $11.051,00$ \\
\hline P 30 & $1.265,38$ & $1.265,38$ & $1.328,18$ & 0,94 & 1,04 & 1,15 & $19.987,19$ & $-16.813,00$ & $-7.938,45$ & $7.809,00$ \\
\hline P 5715 & $1.145,18$ & $1.145,18$ & $1.192,83$ & 0,86 & 0,94 & 1,02 & $11.577,30$ & $-21.931,00$ & $-16.348,33$ & $-5.951,00$ \\
\hline \multirow[t]{2}{*}{ Alfa 200} & $1.163,06$ & $1.163,06$ & $1.205,36$ & 0,99 & 1,07 & 1,14 & $9.527,26$ & $-22.960,00$ & $-18.398,37$ & $-9.926,00$ \\
\hline & \multicolumn{10}{|c|}{ Prioris não informativas } \\
\hline Monarca SP INTA & $1.139,52$ & $1.188,95$ & $1.238,72$ & 0,95 & 1,04 & 1,13 & $12.731,40$ & $-21.507,00$ & $-15.194,23$ & $-3.202,00$ \\
\hline Crioula & 990,11 & $1.033,28$ & $1.076,74$ & 1,00 & 1,08 & 1,16 & $9.705,82$ & $-23.033,00$ & $-18.219,81$ & $-9.078,00$ \\
\hline 5929 & $1.212,87$ & $1.265,00$ & $1.316,64$ & 0,84 & 0,93 & 1,02 & $13.930,00$ & $-20.903,00$ & $-13.996,05$ & $-875,00$ \\
\hline $58 \mathrm{~N} 58$ & $1.215,40$ & $1.263,73$ & $1.312,39$ & 1,00 & 1,09 & 1,17 & $12.169,78$ & $-21.791,00$ & $-15.755,86$ & $-4.292,00$ \\
\hline Sutter & $1.140,00$ & $1.211,00$ & $1.282,84$ & 0,86 & 0,98 & 1,11 & $26.300,00$ & $-14.665,00$ & $-1.626,35$ & $23.145,00$ \\
\hline Flórida 77 & $1.223,89$ & $1.287,91$ & $1.352,37$ & 1,01 & 1,12 & 1,23 & $21.357,29$ & $-17.157,00$ & $-6.568,35$ & $13.548,00$ \\
\hline WL 516 & 983,10 & $1.330,00$ & $1.679,00$ & 0,09 & 0,70 & 1,32 & $628.800,00$ & $289.083,00$ & $600.870,80$ & $1.193 .575,00$ \\
\hline Maricopa & $1.391,00$ & $1.459,42$ & $1.527,83$ & 0,92 & 1,04 & 1,16 & $24.065,89$ & $-15.791,60$ & $-3.859,74$ & $18.806,30$ \\
\hline Costera SP INTA & $1.292,00$ & $1.357,02$ & $1.422,05$ & 0,99 & 1,11 & 1,22 & $21.735,82$ & $-16.966,00$ & $-6.189,81$ & $14.283,00$ \\
\hline P 30 & $1.210,72$ & $1.259,58$ & $1.308,77$ & 0,95 & 1,04 & 1,12 & $12.436,67$ & $-21.656,00$ & $-15.488,96$ & $-3.774,00$ \\
\hline P 5715 & $1.093,40$ & $1.142,00$ & $1.190,21$ & 0,86 & 0,95 & 1,03 & $12.120,00$ & $-21.814,00$ & $-15.802,33$ & $-4.382,00$ \\
\hline Alfa 200 & $1.123,03$ & $1.165,53$ & $1.208,31$ & 1,00 & 1,07 & 1,15 & $9.406,05$ & $-23.184,00$ & $-18.519,58$ & $-9.659,00$ \\
\hline
\end{tabular}

Tabela 4. Valores obtidos para o fator de Bayes da comparação de modelos com prioris informativas (i) e não informativas (j), para os genótipos em estudo.

\begin{tabular}{lc}
\hline Cultivar & $\mathrm{FB}_{\mathrm{ij}}$ \\
\hline Monarca SP INTA & 22,20 \\
Crioula & 13,90 \\
5 929 & 23,40 \\
58 N 58 & 16,70 \\
Sutter & 23,70 \\
Flórida 77 & 22,80 \\
WL 516 & 20,40 \\
Maricopa & 22,70 \\
Costera SP INTA & 23,10 \\
P 30 & 20,80 \\
P 5715 & 22,00 \\
\hline
\end{tabular}

apresentou fortes evidências a seu favor para todos os genótipos avaliados. O menor valor do fator de Bayes obtido foi de 13,90, ao se considerar a análise do genótipo Crioula, e o maior valor foi de 23,79 para o genótipo Sutter. Embora as estimativas dos parâmetros de adaptabilidade apresentem resultados semelhantes, o fator de Bayes revela que existem evidências de que o uso de prioris informativas fornece resultados mais acurados. Apesar da informação a priori ter-se baseado em apenas três artigos, ela mostrou-se de grande importância para este estudo. Espera-se que, em estudos de adaptabilidade e estabilidade em outras culturas, que possuam maior quantidade de informação disponível para meta-análise, os resultados sejam ainda mais precisos.

\section{Conclusões}

1. A abordagem bayesiana aplicada a estudos de adaptabilidade e estabilidade por meio de modelos de regressão linear simples é eficiente para a classificação dos genótipos. 
2. Com base nos resultados de seleção de modelos via fator de Bayes, o uso de distribuições a priori informativas é superior à qualidade de ajuste às distribuições a priori não informativas.

\section{Referências}

ANNICCHIARICO, P. Cultivar adaptation and recommendation from alfalfa trials in Northern Italy. Journal of Genetics and Plant Breeding, v.4, p.269-278, 1992.

CASSELA, G.; GEORGE, E.I. Explaining the Gibbs sampler. The American Statistician, v.46, p.167-174, 1992.

CRUZ, C.D.; REGAZZI, A.J.; CARNEIRO, P.C.S. Modelos biométricos aplicados ao melhoramento genético. 3.ed. Viçosa: UFV, 2004. 480p.

EBERHART, S.A.; RUSSELL, W.A. Stability parameters for comparing varieties. Crop Science, v.6, p.36-40, 1966.

FERREIRA, R. de P.; BOTREL, M. de A.; RUGGIERI, A.C.; PEREIRA, A.V.; COELHO, A.D.F.; LÉDO, F.J. da S.; CRUZ, C.D. Adaptabilidade e estabilidade de cultivares de alfafa em relação a diferentes épocas de corte. Ciência Rural, v.34, p.265-269, 2004.

GEWEKE, J.Evaluating the accuracy of sampling-based approaches to the calculation of posterior moments. In: BERNARDO, J.M.; BERGER, J.O.; DAVID, A.P.; SMITH, A.F.M. (Ed.). Bayesian statistics. New York: Oxford University, 1992. Cap.4, p.625-631.

HEIDELBERGER, P.; WELCH, P.D. Simulation run length control in the presence of an initial transient. Operations Research Landing, v.31, p.1109-1144, 1983.

JEFFREYS, H. Theory of probability. $3^{\text {rd }}$ ed. Oxford: Claredon, 1961. 447p.

KASS, R.E.; RAFTERY, A.E. Bayes factors. Journal of the American Statistical Association, v.90, p.773-795, 1995.

LÉDO, F.J. da S.; BOTREL, M. de A.; EVANGELISTA, A.R.; VIANA, M.C.M.; PEREIRA, A.V.; SOUZA SOBRINHO, F. de; OLIVEIRA, J.S. e; XAVIER, D.F.; HEINEMANN, A.B. Adaptabilidade e estabilidade de cultivares de alfafa avaliadas em Minas Gerais. Ciência e Agrotecnologia, v.29, p.409-414, 2005.
MIRANDA, G.V.; SOUZA, L.V. de; GUIMARÃES, L.J.M.; NAMORATO, H.; OLIVEIRA, L.R.; SOARES, M.O. Multivariate analyses of genotype $\mathrm{x}$ environment interaction of popcorn. Pesquisa Agropecuária Brasileira, v.44, p.45-50, 2009.

NASCIMENTO, M.; CRUZ, C.D.; CAMPANA, A.C.M.; TOMAZ, R.S.; SALGADO, C.C; FERREIRA, R. de P. Alteração no método centróide de avaliação da adaptabilidade genotípica. Pesquisa Agropecuária Brasileira, v.44, p.263-269, 2009a.

NASCIMENTO, M.; FERREIRA, A.; CAMPANA, A.C.M.; SALGADO, C.C.; CRUZ, C.D. Multiple centroid methodology to analyze genotype adaptability. Crop Breeding and Applied Biotechnology, v.9, p.8-16, 2009b.

NASCIMENTO, M.; FERREIRA, A.; FERRÃO, R.G.; CAMPANA, A.C.M.; BHERING, L.L.; CRUZ, C.D.; FERRÃO, M.A.G.; FONSECA, A.F.A. da. Adaptabilidade e estabilidade via regressão não paramétrica em genótipos de café. Pesquisa Agropecuária Brasileira, v.45, p.41-48, 2010c.

PEREIRA, H.S.; MELO, L.C.; DEL PELOSO, M.J.; FARIA, L.C. de; COSTA, J.G.C. da; DÍAZ, J.L.C.; RAVA, C.A.; WENDLAND, A. Comparação de métodos de análise de adaptabilidade e estabilidade fenotípica em feijoeiro-comum. Pesquisa Agropecuária Brasileira, v.44, p.374-383, 2009.

RAFTERY, A.L.; LEWIS, S.M. Comment: one long run with diagnostics: implementation strategies for Markov chain Monte Carlo. Statistical Science, v.7, p.493-497, 1992.

R DEVELOPMENT CORE TEAM. R: a language and environment for statistical computing. Vienna: R Foundation for Statistical Computing. Available at: $<$ http://www.R- project.org $>$. Accessed on: 10 Jan. 2010.

ROCHA, R.B.; MURO-ABAD, J.I.; ARAÚJO, E.F.; CRUZ, C.D. Avaliação do método centróide para estudo de adaptabilidade ao ambiente de clones de Eucalyptus grandis. Ciência Florestal, v.15, p.255-266, 2005.

VASCONCELOS, E.S. de; BARIONI JÚNIOR, W.; CRUZ, C.D.; FERREIRA, R. de P.; RASSINI, J.B.; VILELA, D. Seleção de genótipos de alfafa pela adaptabilidade e estabilidade da produção de matéria seca. Acta Scientiarum. Agronomy, v.30, p.339-343, 2008.

Recebido em 17 de maio de 2010 e aprovado em 14 de dezembro de 2010 